\title{
REMARKS ON $\gamma$-OPERATIONS INDUCED BY A TOPOLOGY
}

\author{
WON KEUN MiN
}

\begin{abstract}
Császár [3] introduced the notions of $\gamma$-compact and $\gamma$-operation on a topological space. In this paper, we introduce the notions of almost $\Gamma$-compact, $(\gamma, \tau)$-continuous function and $(\gamma, \tau)$-open function defined by $\gamma$-operation on a topological space and investigate some properties for such notions.
\end{abstract}

\section{Introduction and preliminaries}

Let $X$ be a non-empty set with the power set $\exp X$. A function $\gamma: \exp X \rightarrow$ $\exp X$ is said to be monotonic [2] if and only if $A \subseteq B \subseteq X$ implies $\gamma A \subseteq \gamma B$. The collection of all monotonic functions is denoted by $\Gamma(X)$ and the elements of $\Gamma(X)$ are said to be operations. If $\gamma \in \Gamma(X)$, then a set $A \subseteq X$ is said to be $\gamma$-open [2] if $A \subseteq \gamma A$. For $A \subseteq X$, we denote by $i_{\gamma} A$ the union of all $\gamma$-open sets contained in $A$, i.e., the largest $\gamma$-open set contained in $A$. The complement of a $\gamma$-open set is said to be $\gamma$-closed. Any intersection of $\gamma$-closed sets is $\gamma$-closed, and for $A \subseteq X$, we denote by $c_{\gamma} A$ the intersection of all $\gamma$ closed sets containing $A$, i.e. the smallest $\gamma$-closed set containing $A$. If $\tau$ is a topology on $X$ and we write $c$ for closure, $i$ for interior, $s=c i, p=i c, \alpha=i c i$, $\beta=$ cic, then $c, i, s, p, \alpha, \beta$ are all all elements of $\Gamma(X)$ and s-open, $p$-open, $\alpha$-open, $\beta$-open sets are said to be semiopen [5], preopen [6], $\alpha$-open [7], $\beta$-open [1], respectively. Let $\gamma \in \Gamma(X)$ and $\gamma^{\prime} \in \Gamma(Y)$. Then a function $f: X \rightarrow Y$ is said to be $\left(\gamma, \gamma^{\prime}\right)$-continuous [4] if for each $\gamma^{\prime}$-open set $V$ in $Y, f^{-1}(V)$ is $\gamma$-open in $X$. And $f$ is said to be $\left(\gamma, \gamma^{\prime}\right)$-open [4] if for each $\gamma$-open set $A$ in $X, f(A)$ is $\gamma^{\prime}$-open in $Y$. In this paper, we introduce the notions of $(\gamma, \tau)$ continuous function and $(\gamma, \tau)$-open function and investigate characterizations for such functions. We also introduce the notion of almost $\Gamma$-compact defined by $\gamma$-operation on a topological space.

Theorem $1.1([2])$. Let $\gamma \in \Gamma(X)$ and $A \subseteq X$. Then the statements are hold:

(1) Any union of $\gamma$-open sets is $\gamma$-open.

Received November 26, 2009; Revised December 14, 2010.

2010 Mathematics Subject Classification. 54A05.

Key words and phrases. $(\gamma, \tau)$-continuous, $(\gamma, \tau)$-open, $\Gamma$-closed graph, strongly $\Gamma$-closed graph, $\gamma$-compact, almost $\Gamma$-compact. 
(2) $i_{\gamma} A=X-c_{\gamma}(X-A), c_{\gamma} A=X-i_{\gamma}(X-A)$.

\section{2. $(\gamma, \tau)$-continuous function and $(\gamma, \tau)$-open function}

We recall the notion of $\gamma$-operation introduced in [3]: Let $(X, \mu)$ be a topological space, and $\gamma: \exp X \rightarrow \exp X$ a mapping such that

(1) $A \subseteq B \Rightarrow \gamma A \subseteq \gamma B$.

(2) $\gamma \emptyset=\emptyset, \gamma X=X$.

(3) For $A \subseteq X$ and an open set $G \subseteq X, G \cap \gamma A \subseteq \gamma(G \cap A)$.

Now we call the mapping $\gamma$ an associated operation with $\mu$ on $X$. We will denote an associated operation $\gamma$ with $\mu$ by $\gamma_{\mu}$ (simply $\gamma$ ).

According to [2], a set $A \subseteq X$ is said to be $\gamma$-open if and only if $A \subseteq \gamma A$. Let $(X, \mu)$ be a topological space and $\gamma$ an associated operation with $\mu$. Then an open set is always $\gamma$-open [3].

Definition 2.1. Let $(X, \mu)$ and $(Y, \tau)$ be topological spaces and $\gamma$ an associated operation with $\mu$. Then a function $f:(X, \mu) \rightarrow(Y, \tau)$ is $(\gamma, \tau)$-continuous if for every open set $F$ in $Y, f^{-1}(F)$ is $\gamma$-open in $X$.

Remark 2.2. Let $(X, \mu)$ and $(Y, \tau)$ be topological spaces and let $\gamma$ and $\gamma^{\prime}$ associated operations with $\mu$ and $\tau$, respectively. Then the $\left(\gamma, \gamma^{\prime}\right)$-continuous function $f: X \rightarrow Y$ is $(\gamma, \tau)$-continuous if the associated operation $\gamma^{\prime}: \exp Y \rightarrow \exp Y$ is defined by $\gamma^{\prime}=i n t$, where int is the interior in $Y$.

Theorem 2.3. Let $f:(X, \mu) \rightarrow(Y, \tau)$ be a function on topological spaces and $\gamma$ an associated operation with $\mu$. Then $f$ is $(\gamma, \tau)$-continuous if and only if for each $x \in X$ and each open set $V$ containing $f(x)$, there exists a $\gamma$-open set $U$ containing $x$ such that $f(U) \subseteq V$.

Proof. Suppose $f$ is $(\gamma, \tau)$-continuous. Then for each $x \in X$ and each open set $V$ containing $f(x), f^{-1}(V)$ is $\gamma$-open. Now put $U=f^{-1}(V)$, then the $\gamma$-open $U$ satisfies that $x \in U$ and $f(U) \subseteq V$.

For the converse, let $V$ be an open set in $Y$. Then for each $x \in f^{-1}(V)$, by hypothesis, there exists a $\gamma$-open set $U$ containing $x$ such that $U \subseteq f^{-1}(V)$, and so $f^{-1}(V)=\cup U$. By Theorem 1.1(1), $f^{-1}(V)$ is $\gamma$-open.

Theorem 2.4. Let $f:(X, \mu) \rightarrow(Y, \tau)$ be a function on topological spaces and $\gamma$ an associated operation with $\mu$. Then a function $f$ is $(\gamma, \tau)$-continuous if and only if $f^{-1}(\operatorname{int}(B)) \subseteq \gamma f^{-1}(B)$ for $B \subseteq Y$.

Proof. Let $f$ be $(\gamma, \tau)$-continuous and $B \subseteq Y$. Since $f^{-1}(\operatorname{int}(B))$ is $\gamma$-open in $X$ and $\gamma$ is monotonic,

$$
f^{-1}(\operatorname{int}(B)) \subseteq \gamma f^{-1}(\operatorname{int}(B)) \subseteq \gamma f^{-1}(B) .
$$

For the converse, let $B$ be open in $Y$. Then by hypothesis, $f^{-1}(B)=$ $f^{-1}(\operatorname{int}(B)) \subseteq \gamma f^{-1}(B)$, so that $f^{-1}(B)$ is $\gamma$-open in $X$. Hence $f$ is $(\gamma, \tau)$ continuous. 
Theorem 2.5. Let $f:(X, \mu) \rightarrow(Y, \tau)$ be a function on topological spaces and $\gamma$ an associated operation with $\mu$. Then the following are equivalent:

(1) $f$ is $(\gamma, \tau)$-continuous.

(2) For every closed set $F$ in $Y, f^{-1}(F)$ is $\gamma$-closed in $X$.

(3) $f^{-1}(\operatorname{int}(B)) \subseteq i_{\gamma} f^{-1}(B)$ for $B \subseteq Y$.

(4) $c_{\gamma} f^{-1}(B) \subseteq f^{-1}(c l(B))$ for $B \subseteq Y$.

(5) $f\left(c_{\gamma} A\right) \subseteq \operatorname{cl}(f(A))$ for $A \subseteq X$.

Proof. Straightforward.

Definition 2.6. Let $(X, \tau)$ and $(Y, \nu)$ be topological spaces and $\gamma$ an associated operation with $\nu$. Then a function $f:(X, \tau) \rightarrow(Y, \nu)$ is said to be $(\tau, \gamma)$-open if for every open set $G$ in $X, f(G)$ is $\gamma$-open in $Y$.

Remark 2.7. Let $(X, \tau)$ and $(Y, \nu)$ be topological spaces and let $\gamma$ and $\gamma^{\prime}$ associated operations with $\tau$ and $\nu$, respectively. Then the $\left(\gamma, \gamma^{\prime}\right)$-open function $f: X \rightarrow Y$ is $\left(\tau, \gamma^{\prime}\right)$-open if $\gamma: \exp X \rightarrow \exp X$ is a mapping defined by $\gamma=$ int, where int is the interior in $X$.

Theorem 2.8. Let $f:(X, \tau) \rightarrow(Y, \nu)$ be a function on topological spaces and $\gamma$ an associated operation with $\nu$. Then the following equivalent:

(1) $f$ is $(\tau, \gamma)$-open.

(2) $\operatorname{int}\left(f^{-1}(B)\right) \subseteq f^{-1}\left(i_{\gamma} B\right)$ for $B \subseteq Y$.

(3) $f^{-1}\left(c_{\gamma} B\right) \subseteq \operatorname{cl}\left(f^{-1}(B)\right)$ for $B \subseteq Y$.

(4) $f(\operatorname{int}(A)) \subseteq i_{\gamma} f(A)$ for $A \subseteq X$.

Proof. (1) $\Rightarrow(2)$ For $B \subseteq Y$, by (1), $f\left(\operatorname{int}\left(f^{-1}(B)\right)\right)$ is a $\gamma$-open set such that $f\left(\operatorname{int}\left(f^{-1}(B)\right)\right) \subseteq B$. It implies $f\left(\operatorname{int}\left(f^{-1}(B)\right)\right) \subseteq i_{\gamma} B$, and hence $\operatorname{int}\left(f^{-1}(B)\right)$ $\subseteq f^{-1}\left(i_{\gamma} B\right)$.

$(2) \Leftrightarrow(3)$ It follows from Theorem 1.1.

$(2) \Rightarrow(4)$ Obvious.

$(4) \Rightarrow(1)$ Let $A$ be open in $X$; then by (4) $f(A)=f(\operatorname{int}(A)) \subseteq i_{\gamma} f(A)$, and so $f(A)=i_{\gamma} f(A)$. Thus $f(A)$ is $\gamma$-open.

Theorem 2.9. Let a function $f:(X, \tau) \rightarrow(Y, \nu)$ be topological spaces and $\gamma$ an associated operation with $\nu$. Then $f$ is $(\tau, \gamma)$-open if and only if for $A \subseteq X$, $f(\operatorname{int}(A)) \subseteq \gamma f(A)$.

Proof. Suppose that $f$ is $(\tau, \gamma)$-open. Then for $A \subseteq X$, by Theorem 2.8, $f(\operatorname{int}(A)) \subseteq i_{\gamma} f(A)$ and since $i_{\gamma} f(A) \subseteq \gamma i_{\gamma} f(A)$ and $\gamma$ is monotonic, we have

$$
f(\operatorname{int}(A)) \subseteq i_{\gamma} f(A) \subseteq \gamma i_{\gamma} f(A) \subseteq \gamma f(A) .
$$

The converse is obvious.

Definition 2.10. Let $(X, \mu)$ be a topological space and $\gamma$ an associated operation with $\mu$. Then $X$ is $\Gamma-T_{2}$ if for every two distinct points $x$ and $y$ in $X$, there exist two $\gamma$-open sets $U$ and $V$ containing $x$ and $y$, respectively, such that $U \cap V=\emptyset$. 
Definition 2.11. Let $(X, \mu)$ and $(Y, \tau)$ be topological spaces and $\gamma$ an associated operation with $\mu$. A function $f: X \rightarrow Y$ has a $\Gamma_{\tau}$-closed graph (resp. strongly $\Gamma_{\tau}$-closed graph) if for each $(x, y) \in(X \times Y)-G(f)$, there exist a $\gamma$-open set $U$ and an open set $V$ containing $x$ and $y$, respectively, such that $(U \times V) \cap G(f)=\emptyset$ (resp. $(U \times c l(V)) \cap G(f)=\emptyset)$, where $G(f)=\{(x, f(x)): x \in X\}$.

Lemma 2.12. Let $(X, \mu)$ and $(Y, \tau)$ be topological spaces and $\gamma$ an associated operation with $\mu$. A function $f: X \rightarrow Y$ has a $\Gamma_{\tau}$-closed graph (resp. strongly $\Gamma_{\tau}$-closed graph) if for each $(x, y) \notin G(f)$, there exist a $\gamma$-open set $U$ and an open set $V$ containing $x$ and $y$, respectively, such that $f(U) \cap V=\emptyset$ (resp. $f(U) \cap \operatorname{cl}(V)=\emptyset)$.

Theorem 2.13. Let $(X, \mu)$ and $(Y, \tau)$ be topological spaces and $\gamma$ an associated operation with $\mu$. If $f: X \rightarrow Y$ is $(\gamma, \tau)$-continuous and $Y$ is a $T_{2}$ space, then $f$ has a strongly $\Gamma_{\tau}$-closed graph.

Proof. Let $x, y \in(X \times Y)-G(f)$. Then $y \neq f(x)$ and since $Y$ is $T_{2}$, there exist two open sets $U$ and $V$ such that $f(x) \in U, y \in V$ and $U \cap V=\emptyset$ and so $U \cap c l(V)=\emptyset$. Since $f$ is $(\gamma, \tau)$-continuous, by Theorem 2.3 , there exists a $\gamma$-open set $W$ of $x$ such that $f(W) \subset U$, and so $f(W) \cap \operatorname{cl}(V)=\emptyset$. Thus by Lemma $2.12, f$ has a strongly $\Gamma_{\tau}$-closed graph.

Theorem 2.14. Let $(X, \mu)$ and $(Y, \tau)$ be topological spaces and $\gamma$ an associated operation with $\mu$. If $f: X \rightarrow Y$ is a surjective function with a strongly $\Gamma_{\tau}$-closed graph, then $Y$ is $T_{2}$-space.

Proof. Let $y$ and $z$ be distinct points in $Y$. Then there exists an $x \in X$ such that $f(x)=y$. Thus $(x, z) \notin G(f)$, since $f$ has a strongly $\Gamma_{\tau}$-closed graph, there exist a $\gamma$-open set $U$ and an open set $V$ of $x$ and $z$, respectively, such that $f(U) \cap \operatorname{cl}(V)=\emptyset$. This implies $y \notin c l(V)$, so there exists an open set $W$ such that $W \cap V=\emptyset$. Consequently, $Y$ is $T_{2}$.

Theorem 2.15. Let $(X, \mu)$ and $(Y, \tau)$ be topological spaces and $\gamma$ an associated operation with $\mu$. If $f: X \rightarrow Y$ is a $(\gamma, \tau)$-continuous injection with a $\Gamma_{\tau}$-closed graph, then $X$ is $\Gamma-T_{2}$.

Proof. Let $x_{1}$ and $x_{2}$ be two distinct elements in $X$. Then $f\left(x_{1}\right) \neq f\left(x_{2}\right)$. This implies that $\left(x_{1}, f\left(x_{2}\right)\right) \in(X \times Y)-G(f)$. By hypothesis, there exist a $\gamma$-open set $U$ and an open set $V$ of $x_{1}$ and $f\left(x_{2}\right)$, respectively, such that $(U \times V) \cap G(f)=\emptyset$. Since $f$ is $(\gamma, \tau)$-continuous, there exists a $\gamma$-open set $W$ containing $x_{2}$ such that $f(W) \subset V$. Hence $f(W \cap U) \subseteq f(W) \cap f(U)=\emptyset$. Therefore $W \cap U=\emptyset$ and so $X$ is $\Gamma-T_{2}$.

Let $(X, \mu)$ be a topological space and $\gamma$ an associated operation with $\mu$. A collection $\mathbf{S}=\left\{S_{i} \subseteq X: S_{i}\right.$ is $\gamma$-open, $\left.i \in I\right\}$ is called a $\gamma$-open cover for $X$ if $X=\cup_{i \in I} S_{i}$. The space $(X, \mu)$ is said to be $\gamma$-compact (resp. almost $\gamma$-compact) [3] if for each $\gamma$-open cover $\mathbf{S}=\left\{S_{i} \subseteq X: S_{i}\right.$ is $\gamma$-open, $\left.i \in I\right\}$, there exists a 
finite index set $F \subseteq I$ such that $X=\cup_{i \in F} S_{i}$ (resp. $X=\cup_{i \in F} c l\left(S_{i}\right)$ ). And we recall that a topological space $(X, \mu)$ is said to be quasi $H$-closed [8] if for each open cover $\mathbf{C}=\left\{G_{i} \subseteq X: G_{i}\right.$ is open, $\left.i \in I\right\}$, there exists a finite index set $F \subseteq I$ such that $X=\cup_{i \in F} c l\left(G_{i}\right)$.

Definition 2.16. Let $(X, \mu)$ be a topological space and $\gamma$ an associated operation with $\mu$. The space $(X, \mu)$ is said to be almost $\Gamma$-compact if for each $\gamma$-open cover $\mathbf{S}=\left\{S_{i} \subseteq X: S_{i}\right.$ is $\gamma$-open, $\left.i \in I\right\}$, there exists a finite index set $F \subseteq I$ such that $X=\cup_{i \in F} c_{\gamma} S_{i}$.

Remark 2.17. Let $(X, \mu)$ be a topological space and $\gamma$ an associated operation with $\mu$. Since every open set is $\gamma$-open, in general, $c_{\gamma}(A) \subseteq \operatorname{cl}(A)$. So obviously the following implications are obtained but the converses may not be true as shown in the next example.

$$
\gamma \text {-compact } \Rightarrow \text { almost } \Gamma \text {-compact } \Rightarrow \text { almost } \gamma \text {-compact }
$$

Example 2.18. (1) Let $X$ be a topological space and the associated operation $\gamma=$ int in $X$. Then since $\gamma$-compactness is compactness and almost $\Gamma$-compactness (almost $\gamma$-compactness) is quasi $H$-closedness, generally the converse is not true.

(2) Let $N$ denote the set of natural numbers. Consider a topology $\mu=$ $\left\{\emptyset, N_{o}, N_{e}, N\right\}$ where $N_{o}=\{2 n-1: n \in N\}$ and $N_{e}=\{2 n: n \in N\}$. Define $\gamma: \exp N \rightarrow \exp N$ by $\gamma(A)=\operatorname{int}(\operatorname{cl}(A))$ for $A \in \exp (N)$. Then the mapping $\gamma$ is an associated operation with $\mu$ on $N$. Obviously the set of all $\gamma$-open sets is $\exp N$, and so we get the conclusion that the space $N$ is almost $\gamma$-compact but not almost $\Gamma$-compact.

Theorem 2.19. Let $(X, \mu)$ and $(Y, \tau)$ be topological spaces and $\gamma$ an associated operation with $\mu$. Let $f: X \rightarrow Y$ be a surjective $(\gamma, \tau)$-continuous function. Then if $X$ is $\gamma$-compact, then $Y$ is compact.

Proof. Obvious.

Theorem 2.20. Let $(X, \mu)$ and $(Y, \tau)$ be topological spaces and $\gamma$ an associated operation with $\mu$. Let $f: X \rightarrow Y$ be $a(\gamma, \tau)$-continuous and surjective function. If $X$ is almost $\Gamma$-compact, then $Y$ is quasi $H$-closed.

Proof. Let $\mathcal{S}=\left\{S_{i}: i \in J\right\}$ be an open cover of $Y$. Then $\left\{f^{-1}\left(S_{i}\right): S_{i} \in\right.$ $\mathcal{S}, i \in J\}$ is a $\gamma$-open cover of $X$ and by almost $\Gamma$-compactness, there is a finite subcover $\left\{c_{\gamma} f^{-1}\left(S_{j_{1}}\right), c_{\gamma} f^{-1}\left(S_{j_{2}}\right), \ldots, c_{\gamma} f^{-1}\left(S_{j_{n}}\right): S_{j} \in \mathcal{S}, j=j_{1}, j_{2}, \ldots, j_{n}\right\}$ such that $X \subseteq \cup c_{\gamma} f^{-1}\left(S_{j}\right)$. Then from Theorem 2.5(4),

$$
Y=f(X) \subseteq f\left(\cup c_{\gamma} f^{-1}\left(S_{j}\right)\right) \subseteq \cup f\left(f^{-1}\left(c l\left(S_{j}\right)\right)\right) \subseteq \cup c l\left(S_{j}\right) .
$$

Hence $Y$ is quasi $H$-closed. 


\section{References}

[1] M. E. Abd El-Monsef, S. N. El-Deeb, and R. A. Mahmoud, $\beta$-open sets and $\beta$-continuous mapping, Bull. Fac. Sci. Assiut Univ. A 12 (1983), no. 1, 77-90.

[2] A. Császár, Generalized open sets, Acta. Math. Hungar. 75 (1997), no. 1-2, 65-87.

[3] _ _ , $\gamma$-compact spaces, Acta. Math. Hungar. 87 (2000), no. 1-2, 99-107.

[4] — $\gamma$-connected sets, Acta. Math. Hungar. 101 (2003), no. 4, 273-279.

[5] N. Levine, Semi-open sets and semi-continuity in topological spaces, Amer. Math. Monthly 70 (1963), 36-41.

[6] A. S. Mashhour, M. E. Abd El-Monsef, and S. N. El-Deeb, On precontinuous and weak precontinuous mappings, Proc. Math. and Phys. Soc. Egypt 53 (1982), 47-53.

[7] O. Njästad, On some classes of nearly open sets, Pacific J. Math. 15 (1965), 961-970.

[8] N. V. Velicko, H-closed topological spaces, Amer. Math. Soc. Transl. 78 (1968), 103-118.

Department of Mathematics

KANGWON NATIONAL UNIVERSITY

Chuncheon 200-701, Korea

E-mail address: wkmin@kangwon.ac.kr 